\title{
Estudio Correlacional de Factores como Desempleo e Índices de Delincuencia en Ecuador
}

\author{
Felix M. Carrera, Flor K. Govea, Gabriela E. Hurtado y Cesar E. Freire \\ Facultad de Ciencias Económicas y Administrativas, Universidad Católica Santiago de Guayaquil \\ (Correo-e: felix.carrera@cu.ucsg.edu.ec; karina.govea@cu.ucsg.edu.ec; Gabriela.hurtado@cu.ucsg.edu.ec; \\ cesar.freire@cu.ucsg.edu.ec)
}

Recibido Ago. 16, 2018; Aceptado Nov. 5, 2018; Versión final Ene. 2, 2019, Publicado Jun. 2019

\begin{abstract}
Resumen
En el presente estudio se analizaron las variables de desempleo y crimen en el país por medio de un modelo estadístico de dichas variables para analizar el grado de correlación entre estos factores. El número de actos delictivos en Ecuador son preocupantes, en especial si se toma en cuenta la información sobre robos, ya que estos muestran una tendencia creciente. Por esta razón, el desempleo y el crimen se han transformado en un problema primario desde un enfoque social. Además, en la actualidad no ha sido muy estudiado en el Ecuador. Los resultados encontrados recomiendan que el desempleo y el crimen pueden ser estudiados de manera eficiente utilizando una metodología estadística como Anova y el coeficiente de correlación. Este análisis demuestra que el desempleo y el crimen se relacionan, aunque estas variables en ocasiones sean asimétricas. Por último, se pudo evidenciar que, a mayor número de personas desempleadas, el número de actos delictivos en el país es mayor.
\end{abstract}

Palabras clave: desempleo; crimen; delincuencia; anova; estudios de correlación

\section{Correlational Study of Factors such as Unemployment and Crime Indices in Ecuador}

\begin{abstract}
In the present study, the variables of unemployment and crime in the country were analyzed by means of a model to find the degree of correlation of these factors. The number of criminal acts in Ecuador is increasing, especially if the information on robberies is taken into account, since they show a steady growing trend. For this reason, unemployment and crime have become a primary problem from a social perspective. Additionally, the subject has not been studied in Ecuador. The results show that unemployment and crime can be efficiently studied using statistical methodology such as Anova and the correlation coefficient. This analysis shows that unemployment and crime are related although these variables are sometimes asymmetric. Finally, it was possible to demonstrate that greater the number of unemployed people is, greater is the number of criminal acts in the country.
\end{abstract}

Keywords: unemployment; crime; delinquency; anova; correlation studies 


\section{INTRODUCCIÓN}

El objeto de la presente investigación es determinar el nivel de correlación que existe entre factores socioeconómico como el desempleo y la delincuencia en el país Ecuador. Se requiere realizar un análisis minucioso para encontrar resultados contundentes sobre el estudio. El proyecto investigativo servirá para poder comprender los resultados de los movimientos y comportamientos de las variables. Debido a que las cifras del desempleo en Ecuador han ido en constante crecimiento, se plantea un estudio para conocer la incidencia que esta variable tiene con la delincuencia que radica en el país. Esta investigación ha sido implementada en países desarrollados para conocer el comportamiento de los ciudadanos que sufren de escasez de un trabajo estable que brinde todos los beneficios y seguridades. Es por ello la vitalidad de adaptar este estudio en nuestro medio para poder medir la relación existente de estos factores económico-social.

La delincuencia genera elevados gastos y daños tanto en la economía como en la psicología de una persona y a la vez de la comunidad, teniendo la potestad de considerar desde varios puntos de vista establecidos por Detotto y Vannini (2010), Czabanski (2008), Lee y Holoviak (2006) y Lambsdorff (2003). Incluso el Banco Mundial (1997) estableció que tanto el crimen como la violencia afectan significativamente el índice de pobreza de un país, teniendo estas una relación directa. También, las investigaciones realizadas por Brand y Price, los primeros estudios sobre la correlación entre la actividad criminal y económica de la sociedad fueron llevados a cabo por Fleisher (1963) y Becker (1968). Además, existen otros distintos enfoques por parte de Brand y Price (2000), Anderson (1999) y Barro (1996) sobre los factores que son capaces de intervenir en la tipología, los cuales son: el género, el nivel educativo, la raza, las condiciones del entorno físico, la edad, el desempleo y la desigualdad económica o social (Ramírez, 2014). En Ecuador, según el Instituto Nacional de Estadística y Censos (INEC, 2016), "la tasa de desempleo urbano se ubicó en el 6,7\% y en área rural en 2,3\% en septiembre del 2016", lo que nos da lugar para iniciar esta investigación y establecer la relación que conlleva con la delincuencia.

Analizando desde el punto de vista moral y jurídico, Durkheim (1987) manifestó que el crimen debería ser penado debido a que no se encuentra dentro de las normas legales por lo cual tiene un merecimiento sancionatorio. En la delincuencia, existe un vínculo entre la utilidad marginal y el costo, fundamentado en el costo como manera de sanción o apresamiento, estudiándolo desde la perspectiva económica. De acuerdo a Becker (1968) el delincuente es un ser con raciocinio y conocimiento, capaz de tomar sus propias decisiones y poder diferenciar entre el bien y el mal, es capaz de utilizarla para decidir entre actuar en una actividad ilegal y legal. Todo ser pensante tomará en cuenta realizar un acto delictivo o ilegal, sólo en el caso de que obtenga una ganancia mayor que el peligro que corre al momento de ponerlo en marcha. Es por esto que se ve involucrado un agente externo en esa toma de decisión el cual es la política pública. De esto dependerá qué tan seguro será el lugar donde uno se encuentre. Algunos estudios han demostrado que el mayor índice de criminalidad se da en sectores donde los habitantes no solamente poseen un nivel de educación relativamente bajo, sino que también pertenecen a una clase social con deplorables condiciones económicas (Cohen, 1955; Ramírez, 2014). La delincuencia crea dos tipos de daño en la sociedad, siendo estos tantos de manera directa como también indirecta. Los directos provienen de las posibles pérdidas materiales y de vidas a la vez causando esto una baja en la economía, mientras que Sandler y Enders (2008) explicaron que los indirectos pasan por el aumento en los costos que conllevan a brindar seguridad. También se puede realizar una revisión de las humanidades que influyen en el crimen de manera global, estas pueden obtenerse a través del estudio realizado por Buonanno (2014).

Es de suma importancia realizar un diagnóstico sobre las causas de la delincuencia, ya que según Matus (2005) debe de tener una secuencia el estudio de los factores. Siendo así, se podría reconocer el vínculo que existe entre estos. De acuerdo a esta investigación se presenta el estudio de variables como delincuencia y el desempleo, con la finalidad de conocer el vínculo que estas pueden poseer. Según las investigaciones de Ramírez (2014), el desempleo "ha sido uno de los indicadores utilizados con mayor frecuencia para dar cuenta de la relación entre ciclo económico y crimen" (p. 270). Es estudio relacionado con las condiciones económicas y los diferentes tipos de actividades delictivas han permitido tener mayor relevancia con el apoyo de enfoques metodológicos modernos, permitiendo revisar hipótesis inicialmente investigadas por criminólogos a principios del siglo XX (Bonger, 1916; Thomas, 1925). Los estudios recientes en este ámbito indican que durante los episodios de recesión económica, el consiguiente aumento en la tasa de desempleo se convierte en un factor potencialmente detonante de la actividad delictiva; sin embargo, la evidencia empírica internacional dista de ofrecer un consenso en este sentido (Torres, 2016). Con base en las conclusiones de Espinoza-Ramírez (2018) es posible y viable la combinación de las tecnologías móviles, la infraestructura desarrollada en las zonas urbanas en materia de telecomunicaciones y el modelo de crowdsourcing para afrontar los problemas de delincuencia en la Ciudad de México. 
Sin embargo, no basta no realizar estudios empíricos, es necesario también sustentarlo con pruebas teóricas. Pero para que ambas partes concuerden, dependerá siempre de la situación de la región o país que se esté estudiando. El trabajo legal y el delito no necesariamente deben estar relacionados, ya que existen varios trabajos que lo sustentan afirmando que varias personas aun así poseyendo un trabajo legítimo comenten actos ilegales debido a que los ingresos totales del hogar no son suficientes para suplir con los gastos, por lo que recurren al crimen. Existe un listado de investigaciones realizadas en Inglaterra y Estados Unidos de los años noventa, la cual sirve como muestra de que hay una relación inversamente proporcional con la determinante ingresos con lo que respecta a los trabajados lícitos (Farrington, 1994). La medición se concentra en las carencias de la población de las localidades en el acceso a los bienes y servicios básicos, captados en tres dimensiones: educación, vivienda e ingresos, siendo un fenómeno que afecta a las localidades (Marlin et al., 2018).

Se puede sostener que una de las determinantes más influyentes de la delincuencia es el hecho de que las personas que no poseen un currículo limpio de actos ilegales o delictivos se les hacen casi imposible conseguir un trabajo digno. En el trabajo realizado por Bergman (2011) se menciona que comúnmente es una gran barrera para las personas que se encuentran inmersos en el mundo delictivo encontrar un trabajo y en el caso de encontrar uno, relacionarse en ese medio. Esto se debe a que no posee la confianza necesaria para trabajar y desarrollar de la manera adecuada el cargo que desempeñan, debido a que tiene el su subconsciente grabado las malas acciones que han cometido, lo cual no los permite poseer paz interior. Otra de las causas por la que los desempleados que han realizados actos ilegales con anterioridad encuentran dificultades para conseguir un puesto de trabajo honrado y permanente se debe a que los individuos de la sociedad se basan en su pasado para "determinar" las posibles acciones que estos podrían realizar si llegasen a conseguir el puesto de trabajo; es decir, los juzgan a priori y le quitan oportunidades debido al miedo de volver a repetir una mala situación (Olvear, 2005).

Fagan (1992) indicó que principalmente son las opresiones que ejerce el sistema judicial el causante de que los delincuentes se mantengan en estos actos hasta que finalmente terminen abandonando el acto delictivo por lo tan inseguros y desesperados que se sienten. En lo que respecta al punto de vista social hay estudios que sustentan que una vez que se ha sido apresado, causan graves impedimentos al momento de estar en la búsqueda de un trabajo legal, pero si existe algo que es de gran ayuda para evitar estar dentro de estos casos es la pronta experiencia laboral. El autor Ramírez (2014) sostuvo un punto de vista parecido al de Fagan (1992) ya que sus estudios han ayudado a determinar que cuando una persona ya tiene experiencia viviendo en privación, desarrolla sentimientos de frustración que puede expulsarlos hacia las personas de su círculo más cercano, afectando así todas sus relaciones personales, y por ende, encontrará dificultades al momento de querer alcanzar oportunidades que lo desliguen de la disparidad vivida. Cantor y Kenneth (1985) plantearon que hay un nexo doble entre las dos variables establecidas, la cual no es analizada de la manera correcta desde un principio antes del estudio. Se piensa que en el caso en el cual un lugar donde la tasa de desempleo es alta se esperaría que así mismo sea la tasa de delincuencia, y también que al quedarse más tiempo dentro de la casa se sufriría menos el hecho de vivir un asalto o robo. A este segundo hecho se le llama efecto oportunidad, situación que provocaría la extinción de la relación entre ambas variables; es decir, si la mayor parte de individuos desempleados se mantuvieran dentro de sus casas; por ende, la tasa de delincuencia disminuirá. Esta teoría la podríamos comprobar con los datos estadísticos del INEC (2016), en donde se dice que 17 de cada 100 personas han sido víctimas de algún delito; sin embargo, sólo 4 de cada 100 hogares han sido víctimas del robo a la vivienda.

Por otro lado, Greenberg (2001) está en completo desacuerdo con esta teoría, fundamentando que por la parte estadística no es posible establecer una relación notoria entre las variables. Lo cual quiere decir que no siempre será posible reconocer el vínculo, por lo que esto dependerá del entorno en el cual se desarrolle. Los autores Blau y Blau (1982) también determinaron que la variable desempleo no una variable suficiente para determinar si existe o no una variabilidad en el crimen, sino que es necesario incluir el estudio de otras variables demográficas para especificar cuál es la causa específica. Incluso, el autor Neumayer (2003) realizó un estudio de diecisiete años en el que incluyó a 59 países, dicho estudio culminó en el año 1997 y en sus conclusiones no pudo encontrar evidencia de que al aumentar la tasa de desempleo en estos países aumentará el índice del crimen; por lo tanto, determinó que mientras aumentara la muestra estudiada, la relación entre estas variables resultaría cada vez más imperceptible. Pedraza et al., (2012) indican que la solución para esto se enfoca en la generación de fuentes de empleos con los efectos del desarrollo de las TIC. La innovación y posterior implantación de las nuevas tecnologías constituye un factor estratégico para mejorar el acceso a la educación, la competitividad empresarial, impulsar el crecimiento económico y lograr una mayor creación de empleo.

Las variables anteriormente expuestas tienen influencia en la sociedad de un país, debido a que genera mayores gastos públicos en seguridad y contra de la delincuencia, por lo que no produce un efecto positivo en la economía ecuatoriana. Se logra reflejar, que el entendimiento en la relación del desempleo y el crimen 
no siempre queda rotundamente establecida. Se podrá obtener un resultado una vez aplicado el método de estudio con datos que han sido extraídos de la página oficial de datos estadísticos en el Ecuador (INEC, 2017). Acorde a lo planteado, la presente investigación expone un modelo que identificará la relación de las variables anteriormente mencionadas, realizando un énfasis en la variable del desempleo en el país de Ecuador. Además, este estudio representa una innovación en el medio ecuatoriano, y su utilidad parte al introducir un enfoque estadístico fomentado en un modelo que nos indicará el nivel de correlación. Luego de esta introducción se prosigue a la presentación de la metodología implementada en el presente trabajo investigativo. La hipótesis de investigación indica que el desempleo incide positivamente en los índices delictivos.

\section{MÉTODOLOGÍA}

La investigación se considera de diseño no experimental, y de alcance correlacional dado que busca medir relación entre dos variables. El enfoque de la investigación es cuantitativo y este realiza uso de pruebas estadísticas para testear hipótesis. Por otro lado, la lógica de la investigación es deductiva dado que se parte teóricamente de la relación entre desempleo y delincuencia. Los datos de la variable dependiente e independiente de investigación fueron obtenidos de información secundaria proveniente de Instituciones Públicas del Ecuador. La variable desempleo fue obtenido del Instituto Nacional de Estadística y Censos la cual formula este indicador a partir de la Encuesta de empleo que realiza periódicamente este organismo. La variable índice delincuencial fue alimentada conjuntamente entre datos provenientes de las estadísticas del Ministerio del Interior y del Instituto Nacional de Estadística y Censos.

De acuerdo al enfoque metodológico, el presente estudio se encarga de la ejecución y práctica de un modelo. Este modelo analiza el desempleo en el Ecuador (Figura 1) como una variable de relación del nivel de la delincuencia en el país (Figura 2). Para ello es necesario exponer los datos de cada variable, para la visualización íntegra de los datos de los mismos, especificando que las gráficas expuestas son del tipo de modelo autoregresor de series de tiempo que se usan para estudiar la relación causal entre diversas variables que cambian con el tiempo y se influyen entre sí. Desde el punto de vista probabilístico una serie temporal es una sucesión de variables aleatorias indexadas según parámetro creciente con el tiempo. Cuando la esperanza matemática de dichas variables aleatorias es constante o varía de manera cíclica, se dice que la serie es estacionaria y no tiene tendencia secular. Muchas series temporales tienen una tendencia creciente (por ejemplo, el número de automóviles en uso en casi todos los países durante los últimos cincuenta años) o decreciente (por ejemplo, el número de personas que trabajan en la agricultura); otras no tienen tendencia (la luminosidad a horas sucesivas, que varía cíclicamente a lo largo de las 24 horas del día) y son estacionarias. Las series presentadas son datos comprobados mediante pruebas de normalidad, correlación y desestacionalización de la serie como tal, dando así garantía en el uso de los datos.

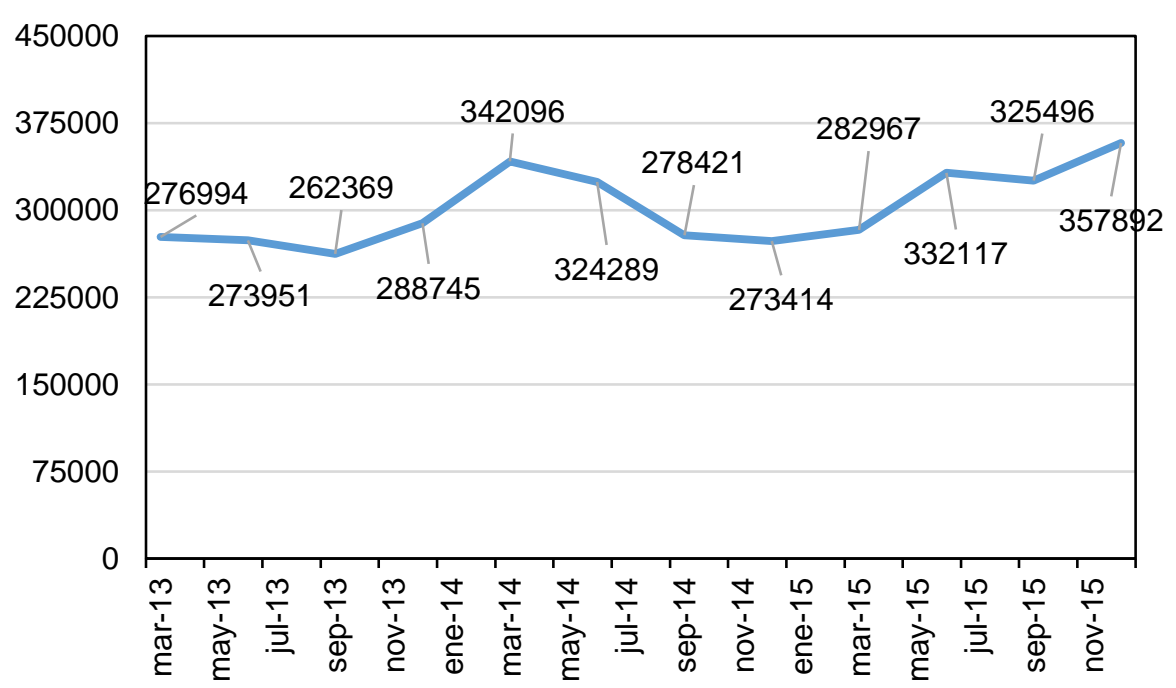

Fig. 1: Desempleo total en Ecuador (2013-2015).

En primera vista figura 1 se muestran los datos de la variable anteriormente mencionada, se puede diferir que los datos guardan cierta relación con la figura 2 que expone la otra variable pero que deben ser analizadas y verificadas. En principio, se confiaría en que con más oportunidades de empleo, mayor número de personas que gocen de inclusión social y de todos los beneficios para satisfacer las necesidades fisiológicas y de una canasta básica podrían llegar a disminuir los casos de crimen existentes año tras año en el Ecuador, siempre y cuando las variables de desempleo y crimen guarden algún tipo de relación. Esta reflexión es dada debido a que estas variables no guardan una correspondencia total de forma, tamaño y posición. 
Es importante atribuir que la delincuencia puede ocasionarse por múltiples factores, como la re inclusión social, la carencia de leyes estatales de prevención u oportunidades de trabajo bien remunerados. Además existen posibilidades de que personas que han sido detenidas por delincuentes, puedan retornar a la vida cotidiana por decisiones tribunales. También es muy probable que personas que han quedado en libertad, retornen nuevamente a la cárcel por una nueva sentencia. Este tipo de pasajes siempre serán un riesgo para toda la sociedad ya que en todo momento estarán expuestas. Cabe recalcar que las personas que cometen actos delictivos siempre mejoran sus habilidades con la práctica y desarrollan nuevas metodologías para favorecer la ejecución de sus habilidades y su propia protección.

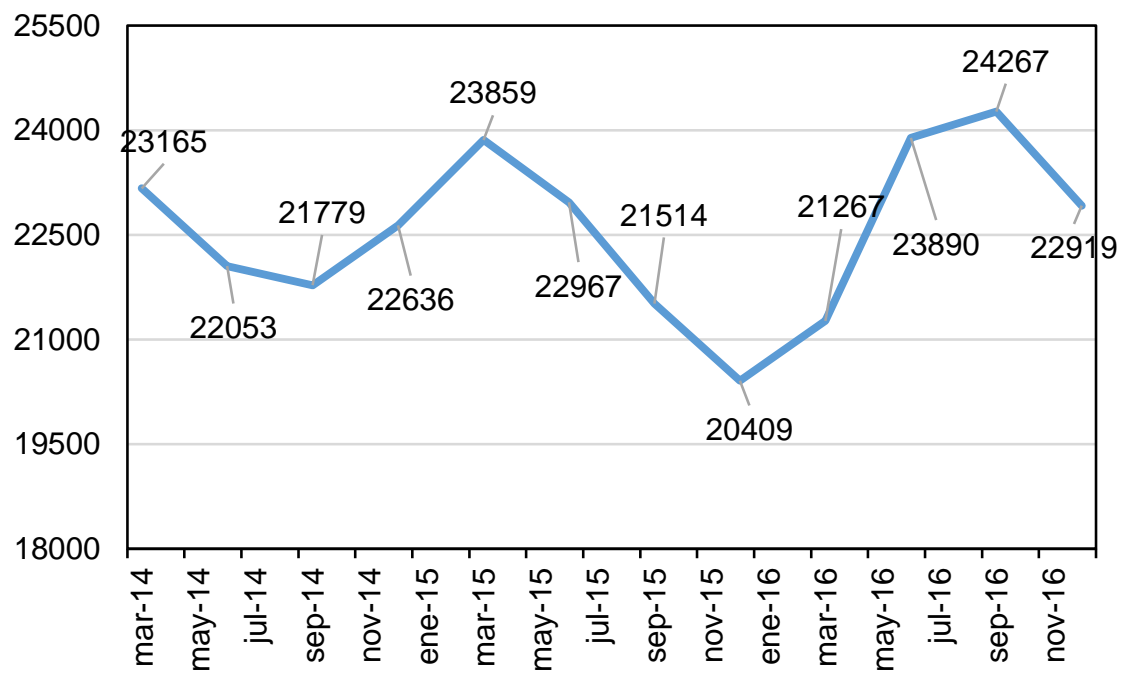

Fig. 2: Delincuencia Total en Ecuador (2014 - 2016).

Por lo tanto se vuelve muy significativo para la policía nacional del Ecuador tener conocimiento de los nuevos pasos o procedimientos de los delincuentes con la finalidad de poder predecir sus comportamientos y facilitar la toma de decisiones policiales y reducir a mayor cantidad el número crimen que existe en el país. Uno de los objetivos de la delincuencia es el estar un paso delante de la ley y esto se logra superando a los policías mediantes varios entornos como lo son: tecnología, conocimiento, armamento y todo recurso que permita llevar a cometer el acto delictivo. Son varios los escritores que indican que la delincuencia y el desempleo no necesariamente tienen una relación que sea directa, y además que la relación podría llegar a ser contradictoria. De acuerdo a esto se debe el extenso debate de esta temática. Es importante agregar que según Nagel (1977); Jacobs (1979) y Braithwaite (1979), existen estructuras estadísticas que nos ayudan a encontrar las relaciones que hay entre el delito y desempleo.

El siguiente estudio correlacional se practica con el objetivo de poder conocer la relación que existe entre el desempleo y la delincuencia, ver como sus resultados tienen alguna variación o influencia sobre la otra variable. Para poder obtener los resultados se implementó la herramienta estadística ANOVA de un factor que permitirá un análisis de la varianza para contrastar la hipótesis nula de que las medias de $n$ poblaciones $(\mathrm{N}>2)$ son iguales, frente a la hipótesis alternativa de que por lo menos una de las poblaciones difiere de las demás en cuanto a su valor esperado. Este contraste es fundamental en el análisis de resultados experimentales, en los que interesa comparar los resultados de $\mathrm{N}$ 'tratamientos' o 'factores' con respecto a la variable dependiente o de interés y un estudio del coeficiente de correlación que es un índice que mide el grado de covariación entre distintas variables relacionadas linealmente. Adviértase que decimos "variables relacionadas linealmente". Esto significa que puede haber variables fuertemente relacionadas, pero no de forma lineal, en cuyo caso no proceder a aplicarse la correlación de Pearson.

Como se indicó anteriormente, se trabajará con información trimestral del año 2013 al 2015 en la variable de desempleo mientras que se ejecutará la relación con la información trimestral del año 2014 al 2016 con la variable delincuencia, se aplica esta relación en el presente estudio debido a que la delincuencia es el efecto o reacción del desempleo, el cual ciertas personas lo practican al no contar con recursos suficientes en un tramo de tiempo, el cual no es inmediato, es decir se espera que el efecto del desempleo genere delincuencia cuatro trimestres posteriores. Se han desarrollado las hipótesis de estudio, las cuales serán comprobadas mediante los resultados obtenidos. La hipótesis nula indica que, a mayor número de desempleo, no se incrementará el número total de casos de delito que existe en el Ecuador, mientras que la hipótesis alternativa, señala que a mayor número de desempleo, se incrementará el número total de casos de delitos que existe en el Ecuador. A continuación como paso final se procederá a realizar los estudios estadísticos para determinar los resultados. 


\section{RESULTADOS}

Las variables que han sido tomadas en cuenta para este análisis son tanto desempleo como crimen, éstas se han realizado con datos trimestrales en el periodo 2013 - 2015 en la variable de desempleo, y se aplica información trimestral en el periodo $2014-2016$ a la variable de crimen. La variable de crimen ha tomado en consideración todos los tipos de crimen registrados en el Instituto Nacional de Estadísticas y Censos (INEC): robos, hurtos, violaciones y asesinatos. A su vez los datos obtenidos del desempleo también fueron encontrados en el INEC y es necesario acotar que las tasas de ambas variables también se disponían en números reales, razón por cual no fue indispensable aplicar fórmula alguna para la obtención de los datos numéricos; acorde a los datos posteriormente expuestos se estimó el modelo.

Tabla 1: Coeficiente de correlación de las variables (desempleo - delincuencia)

\begin{tabular}{lcc}
\hline & Desempleo & Delincuencia \\
\hline $\begin{array}{lc}\text { Desempleo } \\
\text { Crimen }\end{array}$ & 1 & \\
\hline
\end{tabular}

Correspondiente a los datos del resultado expuesto en la tabla 1, se puede complementar a que existe un grado de correlación positiva, lo cual da indicios a poder determinar a que el movimiento o cambio de una variable, tiene influencia sobre la otra. El resultado de este estudio además demuestra a que existe una relación fuerte entre los datos de ambas variables. De manera que es necesario, continuar con el estudio para poder comprobar las hipótesis antes planteadas. En consecuencia el análisis de correlación indica que la fuerza de relación lineal entre el desempleo y los índices de delincuencia es del $71.56 \%$. Este indicador demuestra una relación significativa en términos estadísticos. Los resultados obtenidos son respaldos por el análisis de varianza. En contraste con los datos expuestos en la tabla 2, se pudo determinar la suma de los cuadrados del estudio, y los grados de libertad con la finalidad de identificar el número de tratamientos realizados. Es relevante identificar que el punto crítico de la función es equivalente a 4.3009 mientras que el valor del punto calculado es 886.231 , cifras que nos ayudarán a validar las hipótesis planteadas con anterioridad. Además se estructuró la figura 3 para una mayor comprensión de los datos relevantes.

Tabla 2: Análisis de Varianza de las Variables Desempleo y Delincuencia

\begin{tabular}{|l|c|c|c|c|c|c|}
\hline \multicolumn{1}{|c|}{$\begin{array}{c}\text { Origen de las } \\
\text { variaciones }\end{array}$} & $\begin{array}{c}\text { Suma de } \\
\text { cuadrados }\end{array}$ & $\begin{array}{c}\text { Grados de } \\
\text { libertad }\end{array}$ & $\begin{array}{c}\text { Promedio de los } \\
\text { cuadrados }\end{array}$ & $F$ & $\begin{array}{c}\text { Probabilidad } \\
\text { Valor crítico } \\
\text { para } F\end{array}$ \\
\hline Entre grupos & $4.67053 \mathrm{E}+11$ & 1 & $4.67053 \mathrm{E}+11$ & 886.231 & $2.86526 \mathrm{E}-19$ & 4.3009495 \\
\hline Dentro de los grupos & 11594241710 & 22 & 527010986.8 & & & \\
\hline Total & $4.78647 \mathrm{E}+11$ & 23 & & & & \\
\hline
\end{tabular}

El análisis de varianza representado en la tabla 2 muestra claramente la significancia de la variable desempleo para explicar la delincuencia. Con una Valor P de 2.86526E-19 y considerando un nivel de significancia de 0.05 se rechaza hipótesis nula que indica que las variables no tienen relación significativa, por lo tanto no se rechaza hipótesis que indica significancia de la variable desempleo para explicar la delincuencia. Al ejecutar el análisis de la figura 3, podemos diagnosticar que el valor encontrado de la función el cual es $F=886.231$ es mayor al punto crítico para $(f)=4.3009495$.

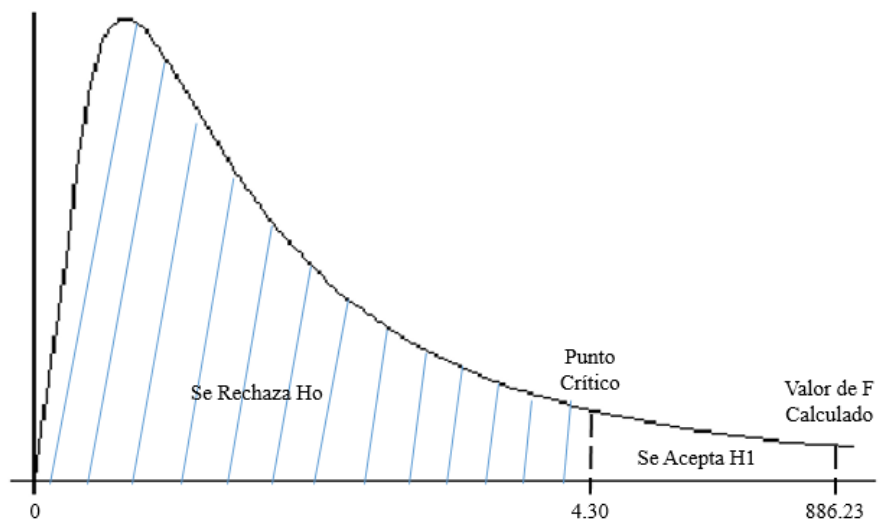

Fig. 3: Diagrama de Prueba de Hipótesis 
El análisis realizado indica que se rechaza la hipótesis nula, y se procede a aprobar la hipótesis alternativa. La hipótesis alternativa señala que a mayor número de desempleo, se incrementará el número total de casos de delitos que existe en el Ecuador. Los resultados obtenidos respaldan evidencia teórica, por lo tanto se puede argumentar una relación causal entre ambas variables, es decir que conforme aumenta el desempleo en un país, los ciudadanos se sienten desesperados ante sus responsabilidades familiares, así como obligaciones con terceros y utilizan como mecanismo de supervivencia acciones delictivas que están fuera del orden de una sociedad.

\section{DISCUSIÓN}

De manera global, lo resultados de esta investigación aparentan ajustarse al análisis de Bergman (2011) la cual realiza una reflexión de la relación concreta que se establecen en las variables del desempleo y el delito. Además aparenta tener cierta discordancia con varias investigaciones internacionales, debido a que los resultados son contradictorios, tal como Andersen (2012) afirmó que estas variables de desempleo y delincuencia no siempre se encuentran relacionadas en un país. Así como también, la investigación de Feliz (2012) representa el primer intento por estudiar sistemáticamente el efecto de la actividad criminal en el crecimiento económico de México. Mediante la estimación de un modelo de convergencia condicional, el autor encuentra que un incremento del diez por ciento en la tasa de homicidios, se asocia con una disminución del 0.13 por ciento en la tasa de crecimiento.

Una crítica importante al modelo utilizado es la aplicación de pruebas paramétricas lo cual puede arriesgar la robustez de los resultados. El uso de pruebas no paramétricas es recomendable para validar los resultados obtenidos bajo otra perspectiva estadística. El presente trabajo investigativo conlleva a un avance en el conocimiento de estas variables a pesar que estas impliquen un grado elevado de complejidad en su comportamiento, acorde a su relevancia del presente, es necesario implementar nuevas investigaciones que indaguen diferentes perspectivas y metodologías que lleven al desarrollo de la dirección establecida por este trabajo. Futuras investigaciones pueden dirigirse a la aplicación de diferentes técnicas estadísticas, por otro lado se puede usar datos de corte transversal considerando totalidad de países registrados en el Banco Mundial.

\section{CONCLUSIONES}

La evolución de datos registrados entre crimen y desempleo denota un crecimiento a manera consecutiva, evidencia de esto es la revelación de los históricos tanto por parte de las denuncias de la policía nacional y el INEC (Instituto Ecuatoriano de Estadística y Censo) sigue siendo uno de las principales características más frecuentadas en dichas instituciones públicas.

Al hacer el análisis de correlación se mostró con evidencia estadística contundente de que hay una alta correlación entre las variables de crimen con el desempleo pues alcanzan un alto valor de relación de 0,72 $\left(R^{2}\right)$. El valor del $R^{2}$ garantiza la calidad de los datos estudiados pues muestra una alta confiabilidad excluyendo sesgo y estocasticidad estadística. Al realizar el análisis de varianza (ANOVA) de las dos variables estudiadas se identificó el rechazo de la hipótesis nula $\left(\mathrm{H}_{0}\right)$ la cual era la nulidad de relación entre ambas variables, quedando la aceptación de la hipótesis alternativa $\left(\mathrm{H}_{1}\right)$ la cual nos indica que hay una alta relación entre el delito y el desempleo por ende se puede predecir que a mayor desempleo en el Ecuador, sus índices de delincuencia irán en aumento.

En general, los resultados extraídos del estudio logran comprobar algunas teorías en cuanto a la relación del desempleo y el crimen en el Ecuador, a su vez con estudios científicos internacionales similares. Aunque el presente análisis conjetura un avance en la información de las relaciones de desempleo y crimen estudiadas en Ecuador, es evidente que las relaciones causales entre las mismas tienen un fuerte grado de complejidad. Por la riqueza de la presente temática, desde un enfoque judicial y político, se aguarda por nuevas investigaciones que exploren diferentes enfoques y metodologías que conlleven a continuar en la misma dirección establecida por este estudio.

\section{REFERENCIAS}

Andersen, S.H., Unemployment and crime: Experimental evidence of the causal effects of intensified ALMPs on crime rates among unemployed individuals, University Press of Southern Denmark, 1-23 (2012)

Anderson, D., The Aggregate Burden of Crime, Journal of Law and Economics, 611-642 (1999)

Barro, R., Democracy and growth, Journal of Economic Growth, 1-27 (1996)

Becker, G., Crime and Punishment: An Economic Approach, Journal of Political Economy, 166-219 (1968)

Bergman, M., Crimen y desempleo en México (2011) 
Bergman, M., Crimen y desempleo en México: ¿Una correlación espuria? CIDE, 1-27 (2011)

Blau, P. y J. Blau, The Cost of Inequality: Metropolitan Structure, American Sociological Review, 47(1) (1982)

Bonger, W., Criminality and economic conditions, Boston, USA, Little, Brown and Company (1916)

Braithwaite, J., Inequality, Crime and Public Policy, Londres, Routledge and Kegan Paul (1979)

Brand, S. y R. Price, The economic and social costs of crime, Home Office, 1-23 (2000)

Buonanno, P., L. Fergusson y J. Vargas, The Crime Kuznets Curve, Springer, 1-30 (2014)

Cantor, D. y C. Kenneth, Nemployment and crime rates in the post World War II United States: A theoretical and empirical analysis, American Sociological Review, 315-330 (1985)

Cohen, A., Delinquent Boys: The Culture of the Gang, Glencoe (1955)

Czabanski, J., Estimates of cost of crime: History, methodologies, and implications, Warszawa, Springer (2008)

Detotto, C. y M. Vannini, Counting the cost of crime in Italy, Global Crime, 419-438 (2010)

Durkheim, É., La división del Trabajo Social, Madrid, Ediciones Akal (1987)

Espinoza-Ramírez, A., M. Nakano, G. Sanchéz-Pérez y A. Arista-Jalife, Sistemas de Información Geográfica y su Análisis Aplicado en Zonas de Delincuencia en la Ciudad de México, México, Información Tecnológica (2018)

Fagan, J., Drug selling and Licit Income in Distressed Neighborhoods: The Economic Lives of Street-Level Drug Users and Dealers, Urban Institute Press (1992)

Farrington, B., Human Development and Criminal Careers, The Oxford Handbook of Criminology, 510-580 (1994)

Feliz, R., Crimen y crecimiento económico en México. Los estados federales en el periodo 2003-2010. En Aguilar, J. (Coordinador), Las bases sociales del crimen organizado y la violencia en México, 220-245, México: CIES y SSP (2012)

Fleisher, B., The effect of unemployment on juvenile delinquency, The Journal of Political Economy, $539-557$ (1963)

Greenberg, D., Time series analysis of crime rates, Journal of Quantitative Criminal, 290-325 (2001)

INEC, INEC publica cifras del mercado laboral de septiembre 2016, Retrieved from Ecuador en cifras (2016)

INEC, Instituto Nacional de Estadísticas y Censos, Ecuador (2016)

INEC, Instituto Nacional de estadísticas y Censos, Retrieved from Ecuador en cifras (2017)

Jacobs, D., Inequality and Police Strength: Conflict Theory and Coercive Control in Metropolitan Areas, American Sociological Review, 913-925 (1979)

Lambsdorff, J., How Corruption Affects Productivity, Kylos, 452-484 (2003)

Lee, D. y S. Holoviak, Unemployment and Crime: An empirical investigation, Applied Economic Letters, $802-812$ (2006)

Marlin A., A. Solano, P. Choles y R. Cuesta, Caracterización Socioeconómica de la Comunidad Indígena Wayuu de Manzana en Colombia: Un aporte desde la Ingeniería Social, Manzana, Información Tecnológica (2018)

Matus, J., ¿Por qué no bajan las tasas de criminalidad en Chile? Revista de Derecho de la Pontificia Universidad Católica de Valparaíso, 64-95 (2005)

Nagel, J., Crime and Incarceration: A Reanalysis, Fels Discussion. University of Pennsylvania School of Public and Urban Policy United States of America, 20-42 (1977)

Neumayer, E., Is Inequality really a Major Cause of Violent Crime? Evidence From a Cross-National. Escuela de Ciencias Políticas y Económicas de Londres (2003)

Olvear, D., La criminalidad y sus determinantes, Población y Desarrollo (2005)

Pedraza, L., D. López y J. Salcedo, Brecha Digital por Estatus Socio-Económico en la Localidad de Ciudad Bolívar de Bogotá, Colombia, Información Tecnológica (2012)

Ramírez, L, Crimen y economía: una revisión crítica de las explicaciones económicas del crimen. (74). Uam-Xochimilco, México, Abril (2014)

Sandler, T. y W. Enders, Economic consequences of terrorism in developed and developing countries: An overview, Cambridge University Press, 12-59 (2008)

The World Bank, Crime and violence as development issues in Latin America and the Caribbean, Rio de Janeiro (1997) Thomas, D., Social aspects of the business cycles, New York, Routledge and Sons (1925)

Torres, V., Desempleo y criminalidad en los estados de la frontera norte de México: un enfoque espacial bayesiano de vectores auto-regresivos, Ciudad de México, Ensayos Revista de Economía (2016) 